\title{
Results from the Biosurveillance Technical Opportunity Prioritization Survey
}

\author{
Tera Reynolds*1, Bill Storm², Andrew Fine ${ }^{3}$, Howard Burkom ${ }^{4}$, Todd Stout ${ }^{5}$ and Victor Del \\ Rio Vilas ${ }^{6}$
}

${ }^{1}$ International Society for Disease Surveillance, Boston, MA, USA; ${ }^{2}$ Ohio Department of Health, Columbus, OH, USA; ${ }^{3}$ Boston Children's Hospital, Boston, MA, USA; ' Johns Hopkins Applied Physics Laboratory, Laurel, MD, USA; ${ }^{5}$ FirstWatch Solutions, Inc. Encinitas, CA, USA; ${ }^{\circ}$ Pan American Health Organization, Rio de Janeiro, Brazil

\section{Objective}

The objective of the '2013 Biosurveillance Technical Opportunity Prioritization Survey' (Survey) is to gather input from the ISDS community on the current landscape and prioritization of data sources and analytical issues in the field of biosurveillance.

\section{Introduction}

The International Society for Disease Surveillance (ISDS) community comprises a large pool of global expertise. Essential to the ISDS mission of advancing the science and practice of disease surveillance is understanding and setting priorities for research and best practices in public health monitoring. To this end, an ISDS workgroup developed an online survey to identify and prioritize the technical and policy issues of the ISDS community. Through analysis, the Survey will identify respondents' perceptions of opportunities in the area of analytical methodologies.

\section{Methods}

A small, but diverse group of subject matter experts developed the Survey through an iterative process. The final Survey will be deployed online and through e-mail to the ISDS community in October 2013. The results will be analyzed prior to the ISDS Conference.

The pool of questions covers identification of issues, and their prioritization, as well as the allocation of weights to related technical criteria or attributes of importance to biosurveillance.

\section{Results}

Results, both frequencies of responses and prioritization, for all respondents and by strata depending on demographic variables, will be available during the presentation.

\section{Conclusions}

The results of this survey will provide necessary information to assist in understanding and setting priorities for research and best practices in public health monitoring.

\section{Keywords}

Prioritization; Community assessment; Biosurveillance opportunities

\section{Acknowledgments}

This work is supported by the International Society for Disease Surveillance.

*Tera Reynolds

E-mail: treynolds@syndromic.org 